\title{
Regional lymph node involvement is associated with poorer survivorship in patients with upper extremity osteosarcoma than with lower extremity osteosarcoma: a SEER analysis
}

\author{
Xianbiao Xie ${ }^{1,2 \#}$, Ziliang Zeng ${ }^{1,2 \#}$, Jian Tu ${ }^{1,2}$, Hao Yao ${ }^{1,2}$, Yiying Bian ${ }^{1,2}$, Qinglin Jin ${ }^{1,2}$, Dongming $\mathbf{L v}^{1,2}$, \\ Bo Wang ${ }^{1,2}$, Jingnan Shen ${ }^{1,2}$ \\ ${ }^{1}$ Department of Musculoskeletal Oncology Center, The First Affiliated Hospital of Sun Yat-sen University, Guangzhou, China; ${ }^{2}$ Guangdong \\ Provincial Key Laboratory of Orthopedics and Traumatology, Guangzhou, China \\ Contributions: (I) Conception and design: X Xie, Z Zeng; (II) Administrative support: B Wang, J Shen; (III) Provision of study materials or patients: H \\ Yao; (IV) Collection and assembly of data: J Tu, Y Bian; (V) Data analysis and interpretation: Q Jin, D Lv; (VI) Manuscript writing: All authors; (VII) \\ Final approval of manuscript: All authors. \\ "These authors contributed equally to this work. \\ Correspondence to: Bo Wang; Jingnan Shen. Department of Musculoskeletal Oncology Center, The First Affiliated Hospital of Sun Yat-sen University, \\ Guangzhou, China; Guangdong Provincial Key Laboratory of Orthopedics and Traumatology, Guangzhou, China. Email: wangb45@sysu.edu.cn; \\ shenjn@sysu.edu.cn.
}

Background: Although the upper limb is the second most common site of osteosarcoma, investigations into clinical manifestation differences between upper and lower limb patients are still sporadic. We retrospectively investigated the characteristics of these patients to gain a better understanding of the differences between upper and lower limb osteosarcoma patients.

Methods: This retrospective study involved patients diagnosed with extremity osteosarcoma between 1997 and 2016 collected from the Surveillance, Epidemiology, and End Results (SEER) database. Patient characteristics were analyzed with $t$-tests, rank sum tests and chi-square tests. Log-rank tests were applied to evaluate univariate significance, and Cox hazards models were performed in multivariate analysis. A binary logistics regression model was used to screen the risk factors related to lymph node involvement.

Results: In total, 1,882 patients, 1,588 (84.4\%) with lower limb lesions and 294 (15.6\%) with upper limb lesions were enrolled in our study. The patients with upper limb osteosarcoma exhibited poorer 5-year overall survival (OS) than patients with lower limb osteosarcoma (54.8\% vs. 63.2\%, P=0.02). The upper limb patients had more lymph node involvement $(5.6 \%$ vs. $2.7 \%, \mathrm{P}=0.03)$, which was found to be an independent prognostic factor $(\mathrm{P}=0.000)$. Tumors located in the upper limbs and the presence of distal metastasis were risk factors related to lymph node involvement in the extremity $(\mathrm{P}<0.05)$. The upper limb patients were tended to suffer greater risk of being affected by both metastasis and lymph node involvement $(15.7 \%$ vs. $9.4 \%, \mathrm{P}=0.18)$.

Conclusions: Upper limb osteosarcoma patients are characterized by more lymph node involvement than lower limb patients, leading to poorer OS. In addition, upper limb patients are at greater risk for both lymph node involvement and distal metastasis. Our results suggest that upper limb patients should be screened more thoroughly for regional lymph node involvement.

Keywords: Extremity osteosarcoma; lymph node involvement; upper limb; Surveillance, Epidemiology, and End Results database (SEER database); survival

Submitted May 25, 2020. Accepted for publication Sep 20, 2020.

doi: $10.21037 /$ tcr-20-2187

View this article at: http://dx.doi.org/10.21037/tcr-20-2187 


\section{Introduction}

Osteosarcoma is the most common type of primary bone sarcoma. Current treatment for osteosarcoma consists of surgery and chemotherapy, and the goal of surgery is to complete remove the tumor and preserve as much function as possible $(1,2)$.

The most frequent sites are the metaphyseal areas of the long bones in the extremities, which account for $85 \%$ of all primary sites $(3,4)$. Although the upper limb is the second most common site of osteosarcoma, these lesions are still thought to show similar characteristics to other extremity lesions and thus are rarely reported separately from lower limb patients (4-7). Some studies have reported that the upper limb tumor is more proximal and led to more distal metastasis than the lower limb patients and upper limb patients showed poorer survival than lower limb patients $(8-10)$. These findings indicate that additional research is needed to determine which characteristics are predictors of survival in upper and lower limb osteosarcoma patients in order to provide a systematic understanding of the differences between upper and lower limb osteosarcoma patients and to avoid inadvertently compromising patient outcomes.

One aspect of the different clinical manifestation between upper and lower limb patients was reflect on the different distal metastasis rate and it was associated with poorer survival in osteosarcoma patients (8). Osteosarcoma progressed primarily hematogenously and also sometimes lymphogenously (11). Regional lymph node involvement in upper limb was mainly reported in case report but the prevalence of lymph involvement has not been well defined and their clinical manifestation has not been systematically evaluated and compared with other extremity patients (Figure 1).

Currently, population-based databases have become increasingly relevant for studying clinical manifestation and tumor outcome in bone sarcoma research (12). The Surveillance, Epidemiology, and End Results (SEER) database, covering $28 \%$ of US population, is one of the most often used large national cancer databases (13). The main purpose of this study was to systematically evaluated and compared the clinical manifestation and tumor outcome between the upper and lower limbs osteosarcoma patients based on large dataset in SEER database, thereby refining the regional lymph node involvement prevalence in upper and lower limb patients. We present the following article in accordance with the STROBE reporting checklist (available at http://dx.doi.org/10.21037/tcr-20-2187).

\section{Methods}

\section{Patient population}

This retrospective study included data from extremity osteosarcoma patients extracted from the National Cancer Institute's SEER database from 1997 to 2016 (13). The cases met the following inclusion criteria: (I) patients diagnosed with osteosarcoma with positive histological confirmation (ICD-O-3 histologic type: 9180-9187, 9192-9195); (II) high-grade osteosarcoma, which is poorly differentiated and anaplastic; (III) the primary site of the tumor is C40.2Long bones of lower limb and associated joints or C40.0Long bones: upper limb, scapula, and associated joints with site record of "bones and joints"; and (IV) the year of diagnosis was between 1997 and 2016. Patients were collected and included in our study. The exclusion criteria were as follows: (I) follow-up data were missing, or survival did not exceed 0 day; (II) histological confirmation was obtained from autopsy and the death certificate only; (III) osteosarcoma was not the first primary tumor; and (IV) patients with multiple tumor. The study was conducted in accordance with the Declaration of Helsinki (as revised in 2013).

Patient data were extracted from the database using SEER*Stat version 8.3.6 software (National Cancer Institute, Bethesda, MD, USA) (13). We extracted age, sex, race, primary site, histological type, tumor size, lymph node involvement, distal metastasis, AJCC stage, treatment protocols, and survival time until death or the last follow-up for each patient in the study. The AJCC stage was evaluated with the The AJCC 8th edition staging system for soft tissue sarcoma (14). Age was categorized into youth, adult and elderly patient groups, with cut-off points at 18 and 40 years old (15).

The lymph node involvement is based on "Derived AJCC N, 6th ed. (2004-2015)", "Derived AJCC N, 6th ed. (2004-2015)", "Derived SEER Combined N (2016+)", "Derived SEER Combined N Src (2016+)", "N valuebased on AJCC 3rd (1988-2003)", "Regional nodes positive (1988+)", "CS lymph nodes (2004-2015)”, "CS Reg Node Eval (2004-2015)" and the lost message is recode as "NX". And patients with localized extend of disease are also enrolled in the no lymph node involvement patients. 

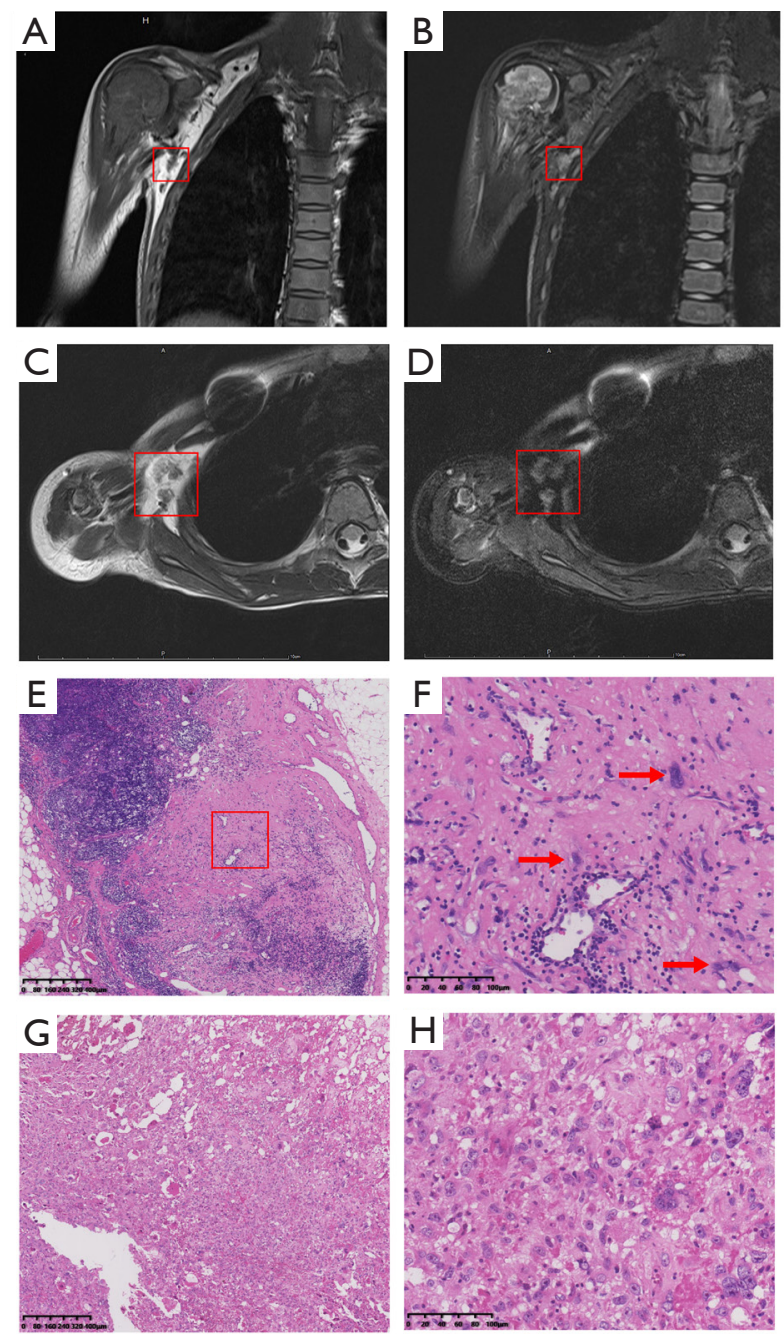

Figure $1 \mathrm{MRI}$ images for axillary node involved osteosarcoma patient. (A) For coronal T1-weighted image for a proximal humeral osteosarcoma patient with axillary lymph node involvement (showed in red box); (B) for coronal T2-weighted image for a proximal humeral osteosarcoma patient with axillary lymph node involvement (showed in red box); (C) for transversal T1-weighted image for a proximal humeral osteosarcoma patient with axillary lymph node involvement (showed in red box); (D) for transversal T2-weighted image (fat suppressed) for a proximal humeral osteosarcoma patient with axillary lymph node involvement (showed in red box); (E) for HE staining ( $\times 80$ scale) of lymph node specimen; $(\mathrm{F})$ for HE staining ( $\times 400$ scale) showing the osteosarcoma cell infiltrated in the lymph node specimen (showed with red arrow); (G) HE staining ( $\times 80$ scale) of primary tumor specimen; $(\mathrm{H}) \mathrm{HE}$ staining ( $\times 400$ scale) of primary tumor specimen.

\section{Statistical analyses}

The demographic and clinical characteristics of enrolled patients were compared using the chi-square test for categorical variables, rank sum tests for grade variables and $t$-tests for continuous variables to determine the baseline differences between the upper and lower limb groups. In the survival analysis, overall survival (OS) was defined as the time from diagnosis to death for patients. Year of diagnosis was dichotomized using optimal cut-off values that were achieved from the time-dependent receiver operating characteristic (t-ROC) curve of the years of diagnosis and patient status at the predicted time (16).

Log-rank tests were applied to evaluate the potential differences between groups in the univariate analysis using the Kaplan-Meier (KM) method. Significant variables $(\mathrm{P}<0.10)$ were included in the multivariate survival analysis using Cox proportional hazards models. The binary logistics regression method was used to evaluate the risk factors related to regional lymph node involvement.

Data were analyzed using SPSS Statistics (version 24.0; IBM, USA), and a t-ROC curve was performed in $\mathrm{R}$ 3.6.2 with the "survival ROC" package with a prediction time of 60 months.

\section{Results}

\section{Demographic and clinical characteristics}

The SEER database contained 2,096 patients who were diagnosed with extremity osteosarcoma and had positive histological confirmation between 1997 and 2016. We excluded 214 patients, and 1,882 patients were ultimately included in our study. The enrolled patients were followed for a median of 45.5 (range: 1-239) months. Among the surviving patients, the median follow-up time was 86 (range: 1-239) months. The flowchart of the patient selection process is reported in Figure 2.

Among the eligible patients, 1,588 (84.4\%) had lower limb lesions, and 294 (15.6\%) had upper limb lesions. Patient characteristics are shown in Table 1 according to the tumor location in the upper and lower limbs. Primary upper limb patients showed similar clinical characteristics with regard to sex, age, race, histologic subtype, tumor size, and distal metastasis ( $\mathrm{P}>0.05$ for all comparisons). However, the upper limb showed more lymph node involvement than the lower limb (5.6\% vs. $2.7 \%, \mathrm{P}=0.03)$. 


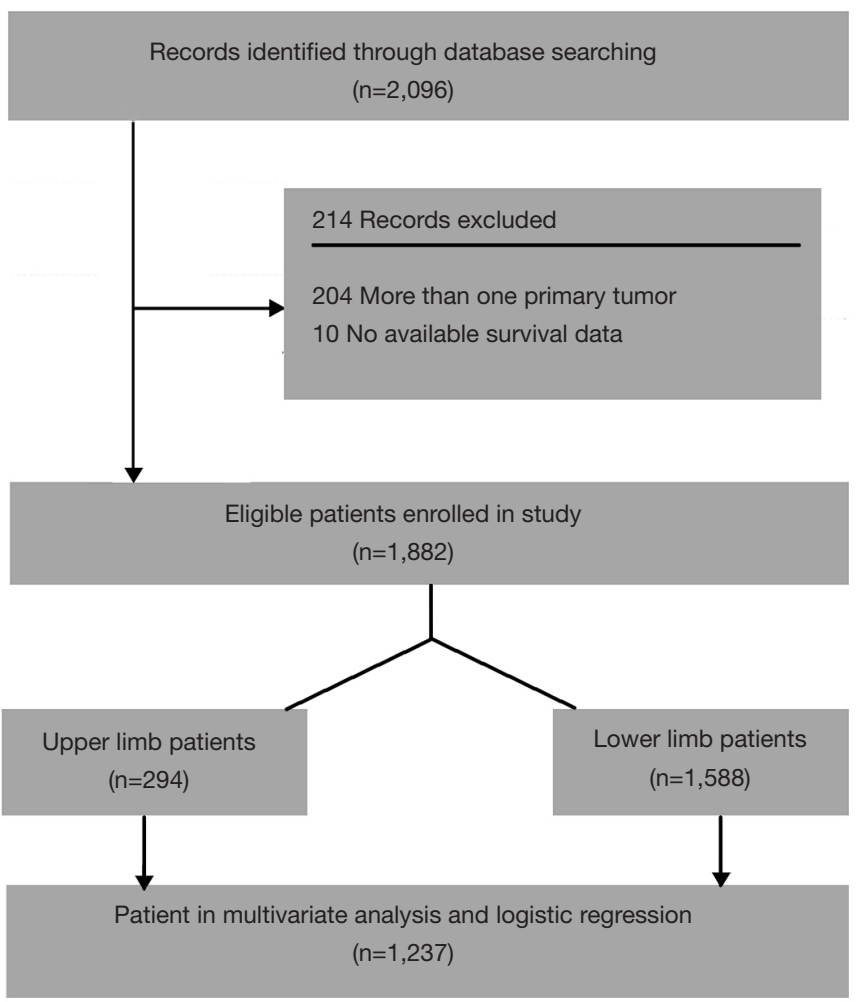

Figure 2 The flowchart describes the study procedure from collect cases from SEER database to statistical analysis. SEER, Surveillance, Epidemiology, and End Results.

The median year of diagnosis was 2009. The upper limb patients were in comparable years of diagnosis with the lower limb patients (median 2008 vs. 2008, $\mathrm{P}=0.07$ ). The t-ROC curve is shown in Figure S1. The Youden index reached its peak in 2011 at 0.037 . However, the AUC for the model was 0.49 , showing that patient survival was not statistically related to the year of diagnosis during 19972016. The KM curves also showed that patient survival remained relatively comparable during this time period $(\mathrm{P}=0.77$, Figure $\mathrm{S} 2)$.

The KM curves of upper and lower limb patients is shown in Figure 3. By the end of follow-up, 683 (36.3\%) of the enrolled patients had died. The 5 -year OS rates of the upper and lower limb groups were $54.8 \%$ and $63.2 \%$, respectively $(\mathrm{P}=0.02)$.

\section{Univariate and multivariate analyses of the prognostic factors}

The results of univariate and multivariate analyses are shown in Table 2. In the univariate analysis, older age, male sex, specific histologic subtype, larger tumor size, more regional lymph node involvement, distal metastasis and disease stage higher than stage I were associated with a worse prognosis $(\mathrm{P}<0.05)$. These prognostic factors were evaluated using Cox proportional hazards models. A total of 1,237 patients with complete data, 1,038 with lesions of the lower limb and 199 with lesions of the upper limb, were enrolled in the multivariate survival analysis. The Cox analysis showed that age $>18$, tumor size $>5 \mathrm{~cm}$, lymph node involvement and distal metastasis were statistically independent variables affecting patients' 5 -year OS $(\mathrm{P}<0.05)$. Their respective KM curves are shown in Figure 4.

\section{Upper limb lesions and distal metastasis were risk factors for lymph node involvement}

The results of the binary logistic regression model of risk factors for lymph node involvement is shown in Table 3. Upper limb lesions and distal metastasis were independent 
Table 1 Characteristics of 1,882 patients with osteosarcoma according to present in upper and lower limbs

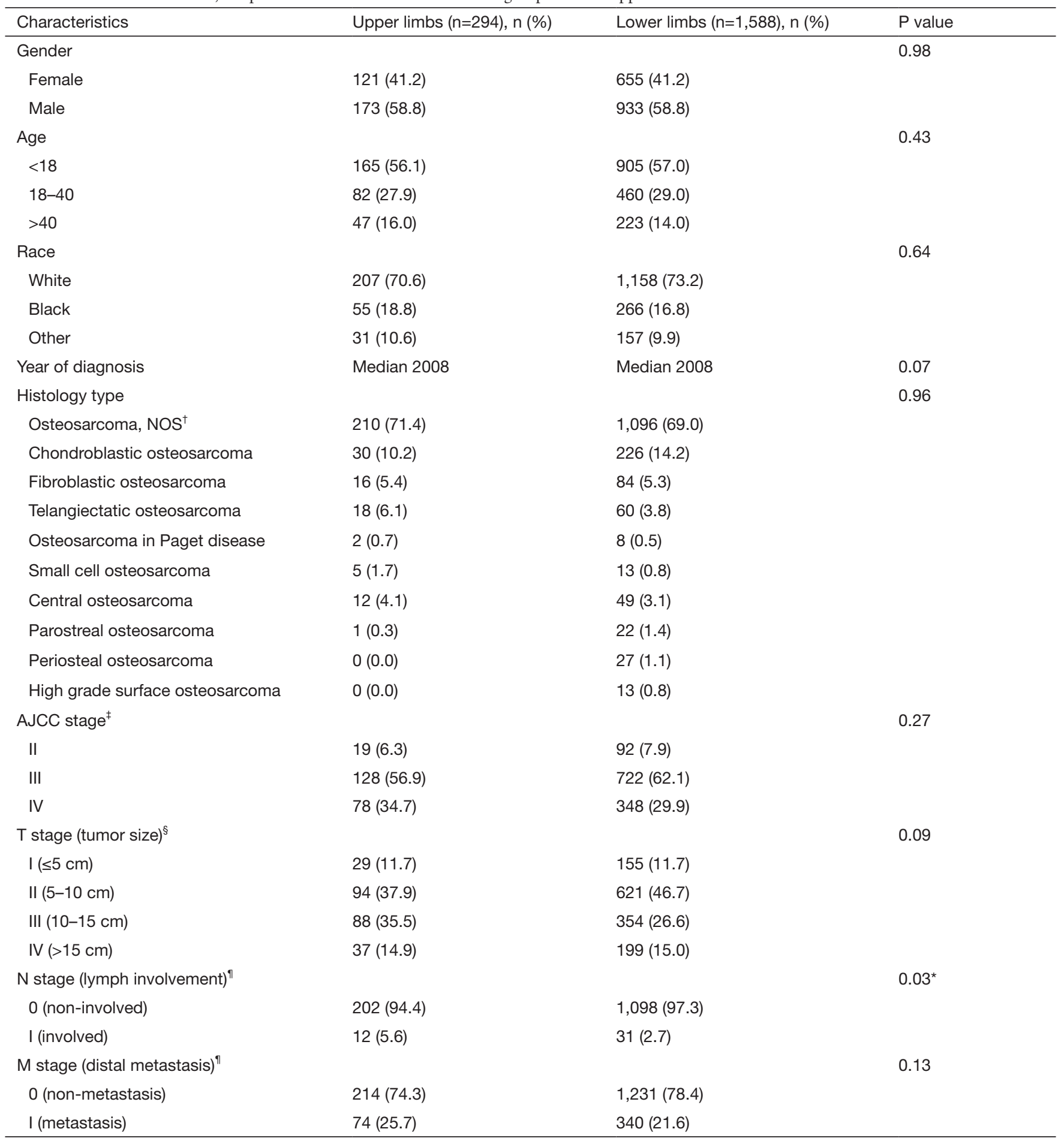

\footnotetext{
${ }^{\dagger}$, NOS is stand for "not otherwise specified" in SEER database; ${ }^{\ddagger}$, AJCC stage is evaluated with The AJCC 8th edition staging system for soft tissue sarcoma; ${ }^{\S}$, tumor size is measured as the maximum diameter of the primary tumor (cm); ", lymph involvement and distal metastasis is diagnosed clinically or pathologically; *, P<0.05. NOS, not otherwise specified; SEER, Surveillance, Epidemiology, and End Results.
} 


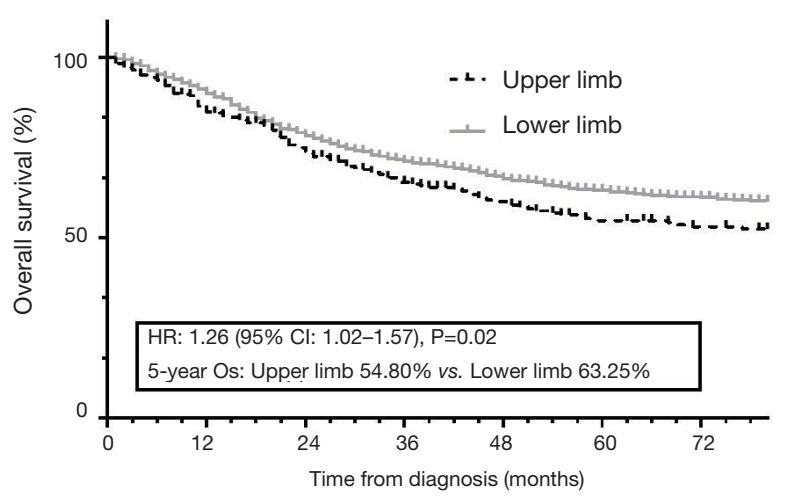

Figure $3 \mathrm{KM}$ curves showed the survival difference between upper and lower limb patients. KM, Kaplan-Meier; OS, overall survival.

risk factors leading to lymph node involvement $(\mathrm{P}<0.05)$, but large tumor size or factors such as age, sex, and histologic type were less related to lymph node involvement $(\mathrm{P}>0.10)$. Although the lymph node-involved histologic subtype remained statistically comparable to no lymph node-involved patients $(\mathrm{P}=0.20)$, fibroblastic osteosarcoma, small cell osteosarcoma, parosteal osteosarcoma, periosteal osteosarcoma and high-grade surface osteosarcoma did not exhibit lymph node involvement $(0.0 \%)$.

A total of $72.1 \%$ (31/43) of lymph node-involved patients also presented with distal metastasis $(\mathrm{P}=0.000)$. Among the metastatic patients, upper limb lesions showed a higher risk of simultaneous lymph and distal involvement, but this difference was not statistically significant $(15.7 \%$ vs. 9.4\%, $\mathrm{P}=0.18$ ). Figure 5 shows the KM curves for subtype analysis among the lymph node-involved and distal metastatic patients. In these progressed patients, the lymph nodeinvolved patients showed a similar 5-year survival to the metastatic patients $(22.9 \%$ vs. $35.8 \%, \mathrm{P}=0.48)$. The patients who were affected with both lymph and distal lesions showed much poorer survival than other progressed patients (5.8\% vs. $35.2 \%, \mathrm{P}=0.000)$.

\section{Discussion}

The results of this study showed that patients with upper limb lesions, which comprise $15.6 \%(294 / 1,882)$ of the total, had a worse 5 -year OS than those with lower limb osteosarcoma $(54.8 \%$ vs. $63.2 \%, \mathrm{P}=0.02)$. This finding is consistent with the results of other studies reporting that upper limb lesions had an unfavorable effect on patient survival compared with other extremity lesions, especially for tumors in the proximal humerus $(4,5)$. However, among existing studies, we found only sporadic investigations into the differences in the clinical manifestation of upper versus lower limb osteosarcoma patients $(1,3,4,8,15,17)$. We found that upper limb patients showed more lymph node involvement $(5.6 \%$ vs. $2.7 \%, \mathrm{P}=0.03)$ than lower limb patients. The prevalence of lymph node involvement is consistent with the present epidemiological study in osteosarcoma (18).

As demonstrated in a previous study, lymph node involvement is an infrequent event in the natural history of osteosarcoma, but it is a highly adverse factor in osteosarcoma patient survival (18-21). We identified that tumors located in the upper limb and distal metastasis are risk factors leading to lymph node involvement in extremity osteosarcoma patients $(\mathrm{P}<0.05)$. It is consistent that some studies have reported that risk location, large tumor size, distal metastasis, high-grade and extraskeletal tumor are important factors to lymph involvement in patients with bone malignance (18,21-24).

Previous studies demonstrated that osteosarcoma could progress not only hematogenously but also lymphogenously in case series (11). Our studies found lymph involvement is often occurred with combination of distal metastasis (72.1\%). It is consistently with previous studies that many case report series observed that lymph node involvement and distal metastasis were occurred simultaneously in extremity osteosarcoma patients (19-21,25). And the result showed the lymph involved patients had no statistical difference in 5-year OS to the metastasis patients (35.8\% vs. $22.9 \%, \mathrm{P}=0.48$ ). It was also reported that regional lymph node-involved patients showed a similarly poor survival like in other metastatic osteosarcoma patients (21). Thus, we postulated that the lymph node involvement presents in a lymphogenous phenotype of distal metastasis and that the difference in lymph node involvement is attributed to the anatomical difference between the regional lymph node network in upper and lower limbs. It can be interpreted as follows: upper limb lesions are most commonly located in the proximal humerus, close to neighboring axial nodes and vessels (26), while lower limb lesions are located near the knee joint, close to popliteal vessels but separated by muscle and interosseous membrane from the lymph network (27).

Our results showed that lymph node involvement is an important prognostic variable that differentiate the tumor outcomes between upper and lower limb osteosarcoma. In patients with no lymph node involvement, the survival differences between upper and lower limb osteosarcoma patients were eliminated $(70.5 \%$ vs. $73.1 \%, \mathrm{P}=0.20)$. This 
Table 2 Univariate and multivariate analyses of clinical characteristics on survival stratified with upper and lower limb

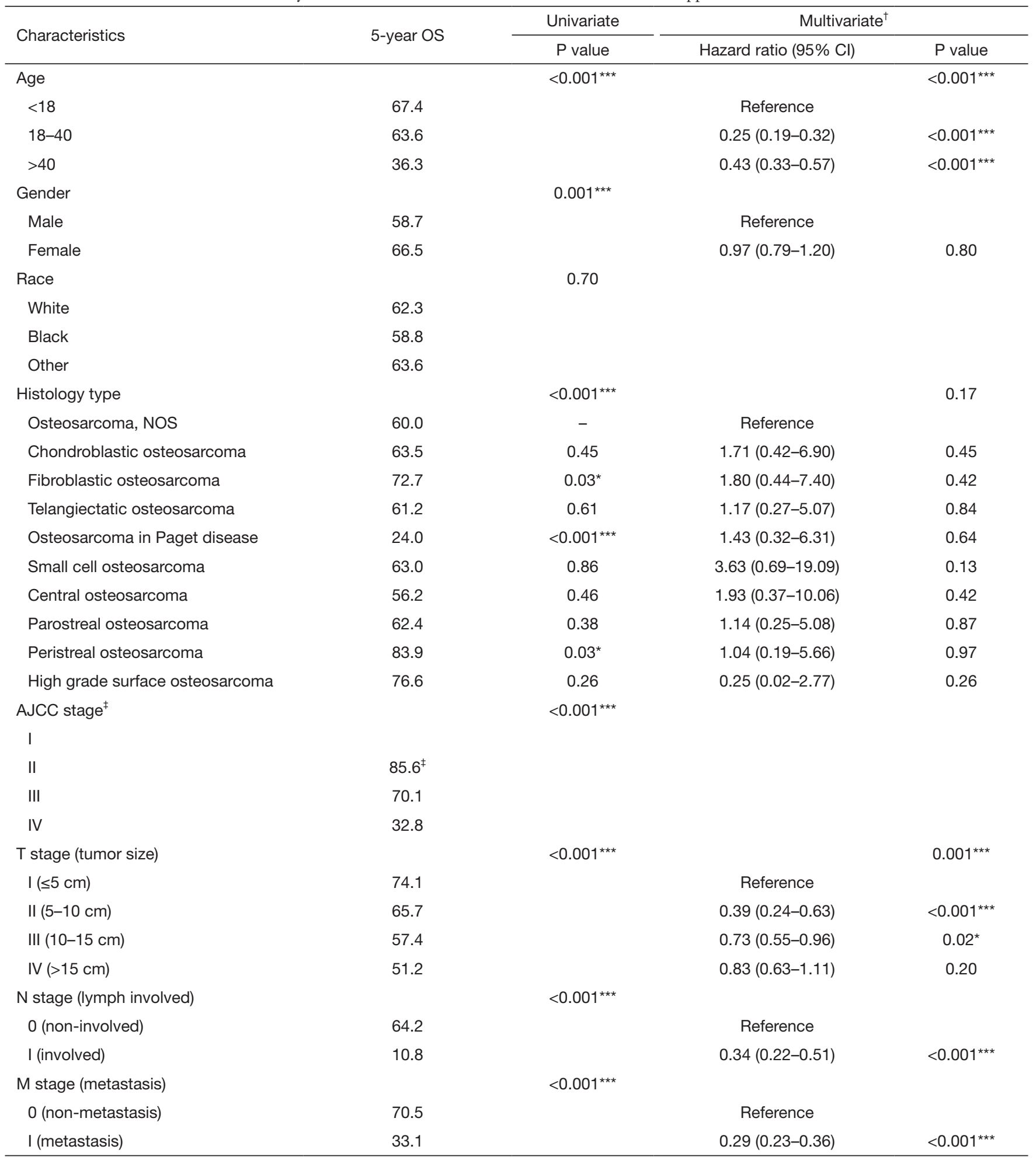

${ }^{\dagger}$, multivariate analysis includes 1,237 cases with complete data, 199 from upper limbs patients and 1,038 from lower limb patients; ${ }^{\ddagger}$, AJCC stage was a composite assessment of tumor size, lymph involved and metastasis and was not included in Cox analysis; *, $\mathrm{P}<0.05 ;{ }^{\star \star \star}, \mathrm{P}<0.001$. OS, overall survival; NOS, not otherwise specified. 
A

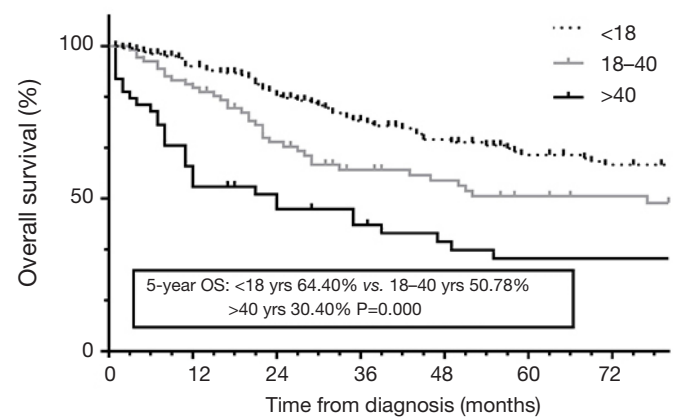

C

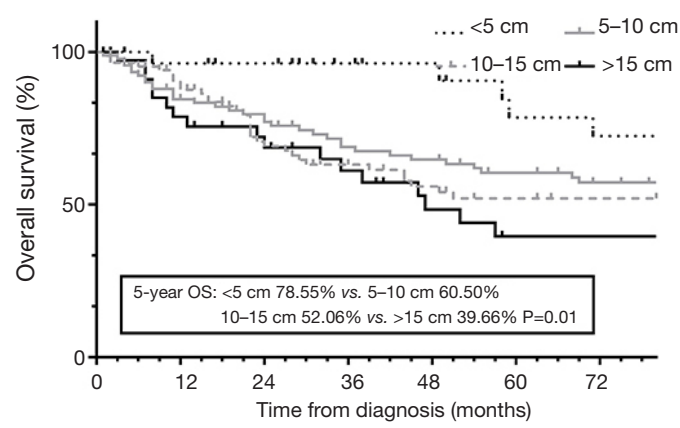

E

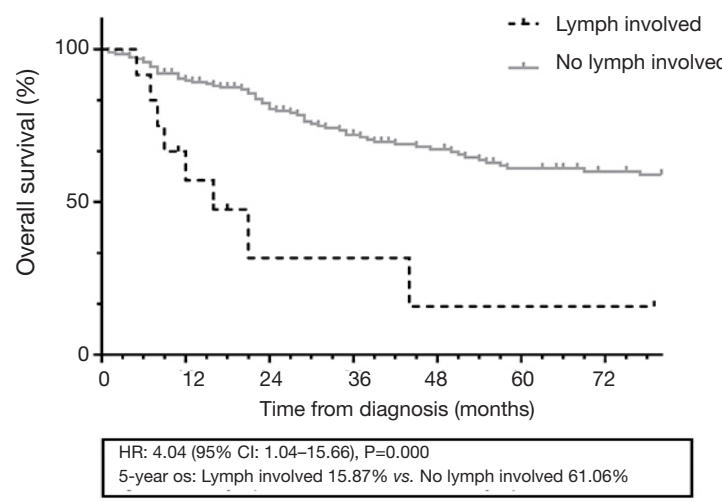

G

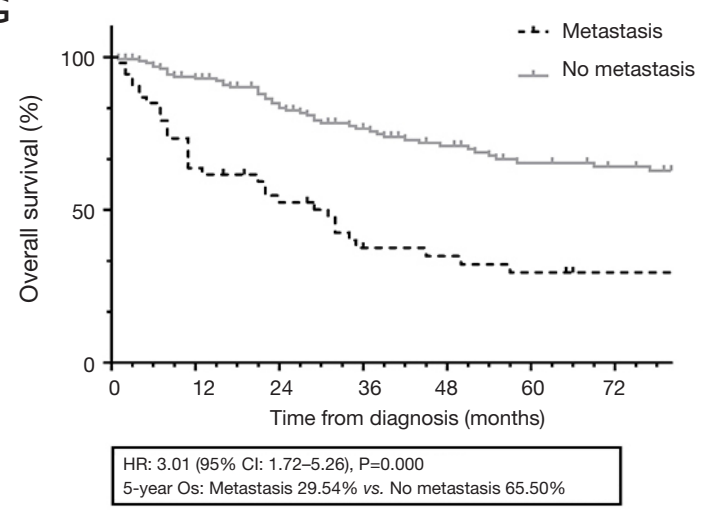

B

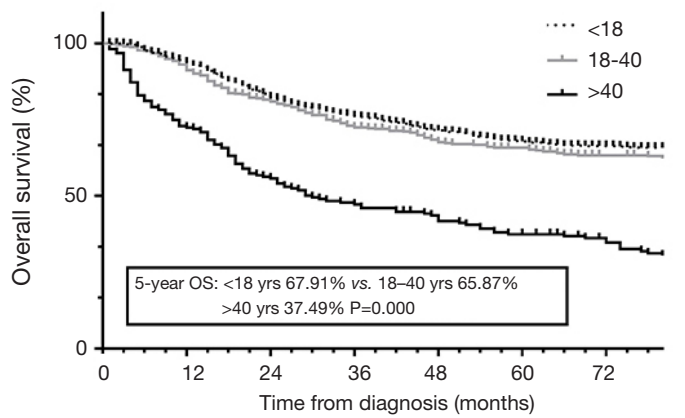

D

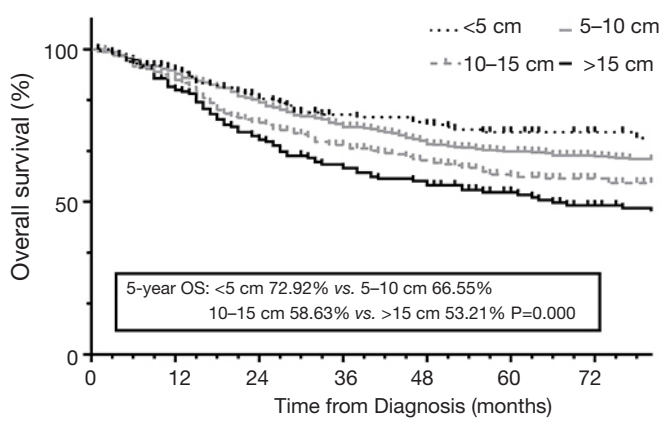

$\mathrm{F}$

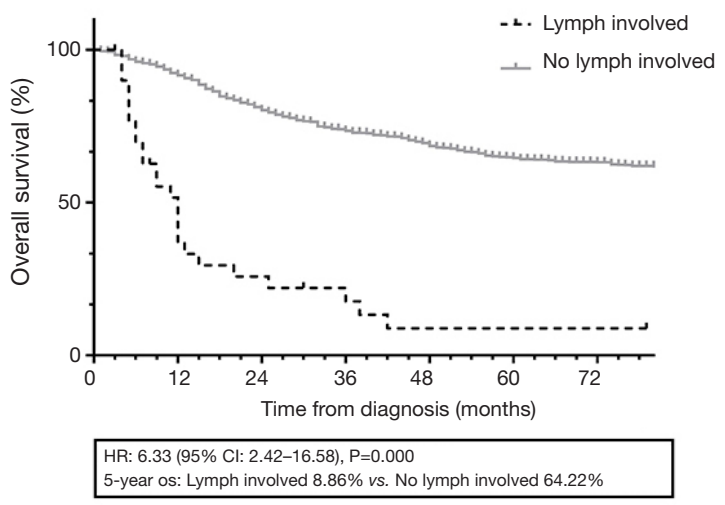

$\mathrm{H}$

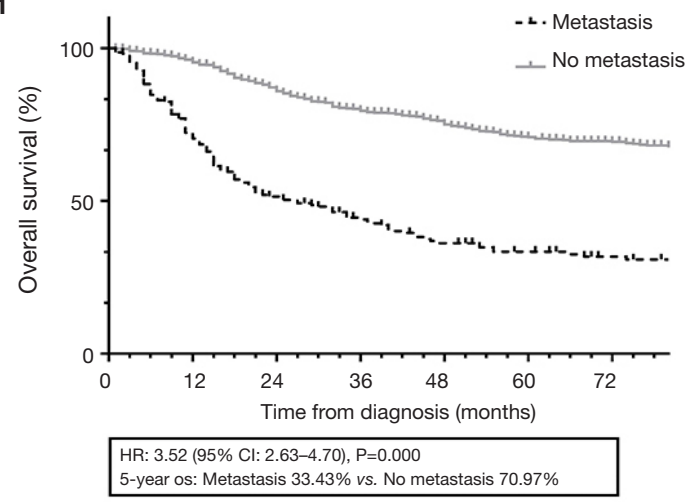

Figure 4 Subgroup KM curves for the prognostic characteristic in upper and lower limb patients. (A) For age subgroup in upper limb patients; (B) for age subgroup in lower limb patients; (C) for tumor size subgroup in lower limb patients; (D) for lymph involvement subgroup in upper limb patients; (E) for lymph involvement subgroup in lower limb patients; (F) for metastasis subgroup in upper limb patients; $(\mathrm{G})$ for metastasis subgroup in lower limb patients; (H) for metastasis subgroup in upper limb patients. KM, Kaplan-Meier; OS, overall survival. 
Table 3 Binary logistics regression of risk factors related to lymph involvement in extremity osteosarcoma

\begin{tabular}{|c|c|c|c|c|}
\hline Characteristics & No lymph involved, n (\%) & Lymph involved, n (\%) & \multicolumn{2}{|c|}{ Binary logistics regression $^{\dagger}$} \\
\hline Age & & & & 0.16 \\
\hline$<18$ & $756(58.2)$ & $22(51.2)$ & Reference & \\
\hline $18-40$ & $357(27.5)$ & $11(25.6)$ & $0.44(0.19-1.02)$ & 0.06 \\
\hline \multicolumn{5}{|l|}{ Gender } \\
\hline Male & $754(58.0)$ & $27(62.8)$ & Reference & \\
\hline Female & $546(42.0)$ & $16(37.2)$ & $0.95(0.47-1.92)$ & 0.88 \\
\hline Race & & & & 0.28 \\
\hline Other & $120(9.3)$ & $169(9.3)$ & $1.41(0.35-5.68)$ & 0.63 \\
\hline Histology type ${ }^{\ddagger}$ & & & & 0.78 \\
\hline Osteosarcoma, NOS & $902(69.4)$ & $33(76.7)$ & Reference & \\
\hline Chondroblastic osteosarcoma & $168(12.9)$ & 7 (16.3) & $1.83(0.23-14.49)$ & 0.57 \\
\hline Fibroblastic osteosarcoma & $60(4.6)$ & $0(0.0)$ & - & - \\
\hline Telangiectatic osteosarcoma & $59(4.5)$ & $1(2.3)$ & $2.25(0.25-20.56)$ & 0.47 \\
\hline Osteosarcoma in Paget disease & $6(0.5)$ & $1(2.3)$ & $1.46(0.08-25.77)$ & 0.80 \\
\hline Small cell osteosarcoma & $13(1.0)$ & $0(0.0)$ & - & - \\
\hline Upper limb & $17(27.9)$ & 286 (15.6) & Reference & \\
\hline Lower limb & $44(72.1)$ & $1,546(84.4)$ & $2.14(1.01-4.55)$ & $0.048^{*}$ \\
\hline T stage (tumor size) & & & & 0.45 \\
\hline $\mathrm{I}(\leq 5 \mathrm{~cm})$ & $116(9.7)$ & $5(13.2)$ & Reference & \\
\hline II (5-10 cm) & $549(45.8)$ & $11(28.9)$ & $1.64(0.48-5.57)$ & 0.43 \\
\hline III $(10-15 \mathrm{~cm})$ & $356(29.7)$ & $13(34.2)$ & $0.65(0.25-1.68)$ & 0.37 \\
\hline $\mathrm{IV}(>15 \mathrm{~cm})$ & $178(14.8)$ & $9(23.7)$ & 0.93 (0.37-2.32) & 0.87 \\
\hline \multicolumn{5}{|l|}{ M stage (metastasis) } \\
\hline 0 (non-metastasis) & 1,032 (79.6) & $12(27.9)$ & Reference & \\
\hline I (metastasis) & 265 (20.4) & $31(72.1)$ & $0.12(0.06-0.25)$ & $<0.001^{\star \star \star}$ \\
\hline
\end{tabular}

${ }^{\dagger}$, binary logistics regression model includes 1,123 cases with complete data, 38 from lymph involved patients and 1,085 from no lymph involved patients; ${ }^{\ddagger}$, the fibroblastic, small cell, parostreal, periosteal, high grade surface osteosarcoma patients were found no lymph involvement and are not included in the logistics regression for the hazard ratio cannot be evaluated; ${ }^{*}, \mathrm{P}<0.05 ;{ }^{* * *}, \mathrm{P}<0.001$. NOS, not otherwise specified. 

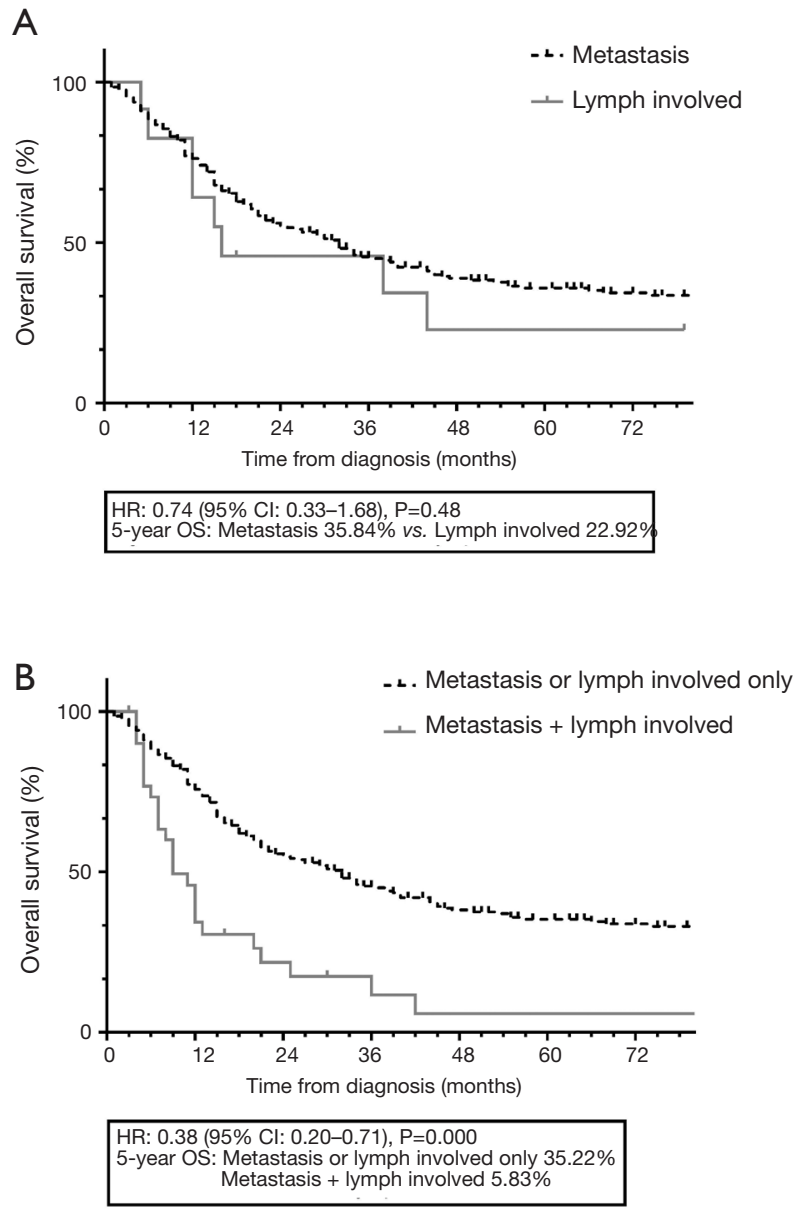

Figure 5 KM curves among the lymph involved and distal metastasis patients. (A) shows similar surviving in lymph involved and distal metastasis patients; (B) shows poorer surviving in patients simultaneously affected with lymph involved and distal metastasis to the lymph involved or distal metastasis only patients. KM, Kaplan-Meier; OS, overall survival.

finding emphasizes the notion that lymph node involvement acts as an independent factor leading to unfavorable survival, especially for upper limb patients. Present diagnostic strategy for lymph node involvement is thorough imaging examinations, including PET-CT and bone scans (28-30). Axillary lymphadenectomy is not primarily recommended to prevent lymph involvement in upper limb osteosarcoma patients for the low prevalence of lymph involvement (30). It suggests that additional work is needed in the future in terms of screening the lymph node status of upper limb osteosarcoma patients.

Nevertheless, our study is limited due to its retrospective nature. First, the SEER database provides trans-sectional data, and thus, we cannot obtain the time axis of patient treatment and disease progression, such as the time of surgery, local recurrence and distal metastasis. These data are useful when considering the time sequence and the causal relation between lymph node involvement and metastasis in the disease. Second, SEER provides lymph node involvement data based on a composite of histologically confirmed regional nodes, clinical and radiographic data and the database has not clarified the diagnosed procedure method in determining lymph node involvement. And the tumor imaging was not available and we are unable to reconfirm the exact regional presentation of the lymph involved osteosarcoma.

Nonetheless, our findings can help physicians better understand extremity osteosarcoma: patients with upper limb lesions tend to have a poorer OS and should be screened more thoroughly to determine regional lymph node involvement like screening the distal metastasis.

\section{Conclusions}

The results of our study demonstrated that upper limb osteosarcoma patients have a worse OS than lower limb osteosarcoma patients. Compared with lower limb patients, upper limb patients are characterized by more lymph node involvement, which is a negative prognostic factor. Upper limb patients have a higher risk of not only lymph node involvement but also distal metastasis. These lymph nodeinvolved patients have similar survival to distal metastatic patients, and a large proportion of lymph node-involved patients are affected by distal metastasis. Our results suggest that upper limb patients should be screened more thoroughly for regional lymph node involvement.

\section{Acknowledgments}

Funding: This work was supported by the National Natural Science Foundation of China $(81202118,81572638$ and 81772864 to $\mathrm{XX})$.

\section{Footnote}

Reporting Checklist: The authors have completed the STROBE reporting checklist. Available at http://dx.doi. org/10.21037/tcr-20-2187

Peer Review File: Available at http://dx.doi.org/10.21037/tcr20-2187 
Conflicts of Interest: All authors have completed the ICMJE uniform disclosure form (available at http://dx.doi. org/10.21037/tcr-20-2187). The authors have no conflicts of interest to declare.

Ethical Statement: The authors are accountable for all aspects of the work in ensuring that questions related to the accuracy or integrity of any part of the work are appropriately investigated and resolved. The study was conducted in accordance with the Declaration of Helsinki (as revised in 2013). This article does not contain any studies with human participants or animals performed by any of the authors.

Open Access Statement: This is an Open Access article distributed in accordance with the Creative Commons Attribution-NonCommercial-NoDerivs 4.0 International License (CC BY-NC-ND 4.0), which permits the noncommercial replication and distribution of the article with the strict proviso that no changes or edits are made and the original work is properly cited (including links to both the formal publication through the relevant DOI and the license). See: https://creativecommons.org/licenses/by-nc-nd/4.0/.

\section{References}

1. Luetke A, Meyers PA, Lewis I, et al. Osteosarcoma treatment - where do we stand? A state of the art review. Cancer Treat Rev 2014;40:523-32.

2. Bielack S, Carrle D, Casali PG, et al. Osteosarcoma: ESMO clinical recommendations for diagnosis, treatment and follow-up. Ann Oncol 2009;20 Suppl 4:137-9.

3. Ottaviani G, Jaffe N. The epidemiology of osteosarcoma. Cancer Treat Res 2009;152:3-13.

4. Bielack SS, Kempf-Bielack B, Delling G, et al. Prognostic factors in high-grade osteosarcoma of the extremities or trunk: an analysis of 1,702 patients treated on neoadjuvant cooperative osteosarcoma study group protocols. J Clin Oncol 2002;20:776-90.

5. Kelley LM, Schlegel M, Hecker-Nolting S, et al. Pathological fracture and prognosis of high-grade osteosarcoma of the extremities: an analysis of 2,847 Consecutive Cooperative Osteosarcoma Study Group (COSS) patients. J Clin Oncol 2020;38:823-33.

6. Pisters PW, Leung DH, Woodruff J, et al. Analysis of prognostic factors in 1,041 patients with localized soft tissue sarcomas of the extremities. J Clin Oncol 1996;14:1679-89.
7. Ferrari S, Bertoni F, Mercuri M, et al. Predictive factors of disease-free survival for non-metastatic osteosarcoma of the extremity: an analysis of 300 patients treated at the Rizzoli Institute. Ann Oncol 2001;12:1145-50.

8. Bramer JA, van Linge JH, Grimer RJ, et al. Prognostic factors in localized extremity osteosarcoma: a systematic review. Eur J Surg Oncol 2009;35:1030-6.

9. Cates JM, Schoenecker JG. Proximal location in extremity long bones is a poor prognostic factor for osteosarcoma: a retrospective cohort study of 153 patients. Acta Oncol 2016;55:1036-9.

10. Cho WH, Song WS, Jeon DG, et al. Differential presentations, clinical courses, and survivals of osteosarcomas of the proximal humerus over other extremity locations. Ann Surg Oncol 2010;17:702-8.

11. Hatori M, Ohtani H, Yamada N, et al. Synchronous multifocal osteosarcoma with lymphatic spread in the lung: an autopsy case report. Jpn J Clin Oncol 2001;31:562-6.

12. Lyu HG, Haider AH, Landman AB, et al. The opportunities and shortcomings of using big data and national databases for sarcoma research. Cancer 2019;125:2926-34.

13. National Cancer Institute. Surveillance, Epidemiology, and End Results Program. 2019. Accessed November 1, 2019. Available online: http://seer.cancer.gov

14. Cates JMM. The AJCC 8th edition staging system for soft tissue sarcoma of the extremities or trunk: a cohort study of the SEER database. J Natl Compr Canc Netw 2018;16:144-52.

15. Song K, Song J, Chen F, et al. Prognostic nomograms for predicting overall and cancer-specific survival of high-grade osteosarcoma patients. J Bone Oncol 2018;13:106-13.

16. Kamarudin AN, Cox T, Kolamunnage-Dona R. Timedependent ROC curve analysis in medical research: current methods and applications. BMC Med Res Methodol 2017;17:53.

17. Bacci G, Briccoli A, Rocca M, et al. Neoadjuvant chemotherapy for osteosarcoma of the extremities with metastases at presentation: recent experience at the Rizzoli Institute in 57 patients treated with cisplatin, doxorubicin, and a high dose of methotrexate and ifosfamide. Ann Oncol 2003;14:1126-34.

18. Thampi S, Matthay KK, Goldsby R, et al. Adverse impact of regional lymph node involvement in osteosarcoma. Eur J Cancer 2013;49:3471-6.

19. Hattori H, Yamamoto K. Lymph node metastasis of osteosarcoma. J Clin Oncol 2012;30:e345-9. 
20. Dirik Y, Cinar A, Yumrukcal F, et al. Popliteal lymph node metastasis of tibial osteoblastic osteosarcoma. Int J Surg Case Rep 2014;5:840-4.

21. Tobias JD, Pratt CB, Parham DM, et al. The significance of calcified regional lymph nodes at the time of diagnosis of osteosarcoma. Orthopedics 1985;8:49-52.

22. Wan L, Tu C, Li S, et al. Regional lymph node involvement is associated with poorer survivorship in patients with chondrosarcoma: a SEER analysis. Clin Orthop Relat Res 2019;477:2508-18.

23. Jacobs AJ, Morris CD, Levin AS. Synovial sarcoma is not associated with a higher risk of lymph node metastasis compared with other soft tissue sarcomas. Clin Orthop Relat Res 2018;476:589-98.

24. Applebaum MA, Goldsby R, Neuhaus J, et al. Clinical features and outcomes in patients with Ewing sarcoma and regional lymph node involvement. Pediatr Blood Cancer 2012;59:617-20.

25. Zwaga T, Bovee JV, Kroon HM. Osteosarcoma of the

Cite this article as: Xie X, Zeng Z, Tu J, Yao H, Bian Y, Jin Q, Lv D, Wang B, Shen J. Regional lymph node involvement is associated with poorer survivorship in patients with upper extremity osteosarcoma than with lower extremity osteosarcoma: a SEER analysis. Transl Cancer Res 2020;9(11):70227033. doi: $10.21037 /$ tcr-20-2187 femur with skip, lymph node, and lung metastases. Radiographics 2008;28:277-83.

26. Kim PW. Variations of the musculofascial axillary arch with the adjacent lymph nodes. Surg Radiol Anat 2020. [Epub ahead of print].

27. Pan WR, Zeng FQ, Wang DG, et al. Perforating and deep lymphatic vessels in the knee region: an anatomical study and clinical implications. ANZ J Surg 2017;87:404-10.

28. Völker T, Denecke T, Steffen I, et al. Positron emission tomography for staging of pediatric sarcoma patients: results of a prospective multicenter trial. J Clin Oncol 2007;25:5435-41.

29. Arkader A, Morris CD. Lymphatic spread of pagetic osteogenic sarcoma detected by bone scan. Cancer Imaging 2008;8:131-4.

30. Wong JC, Abraham JA. Upper extremity considerations for oncologic surgery. Orthop Clin North Am 2014;45:541-64. 